\title{
La mort ou "la migration sans retour"? Une lecture thanatico-psychologique des romans de Maryse Conde
}

\author{
Francis Unimna Angrey
}

Published online: 5 February 2013

(C) The Author(s) 2013. Cet article est publié avec libre accès sur Springerlink.com

Résumé Dans notre communication, nous essayons de démontrer que Maryse Condé, écrivaine guadeloupéenne, fait de la mort un sujet important de discussion dans certains de ses romans. Elle nous y fait voir que la mort peut aussi bien être une source de révolte tout comme elle peut être un élément rassembleur surtout dans une communauté où les gens vivent repliés sur eux-mêmes. En même temps, certains de ses personnages se servent de la mort-suicide comme un moyen de se soustraire à la vie de misère qu'ils mènent. Nous avons décidé de consulter les romans de Condé dont La vie scélérate, Traversée de la mangrove, La migration des cours, Les derniers rois mages, la colonie du nouveau monde pour faire ressortir cette idée que se fait Condé de la mort.

Keywords La mort · Thanatico-psychologique · Romans · Maryse Condé

\section{Introduction}

Dans beaucoup de nos sociétés, même traditionnelles, parler du sujet de la mort en public est un tabou. Malgré ce phénomène, qui pourrait être dû à la peur de la mort, elle reste un fait qui touche toutes les couches sociales de notre société. Les hommes sont partout entourés et couverts par le voile de la mort.

Bien que les hommes n'aiment pas tellement en discuter, la mort est en passe de devenir un sujet important de discussion dans les écrits des écrivains, des artistes et même des critiques littéraires. Pour certains de ces écrivains, la mort est une sorte de "migration sans retour"; pour d'autres, elle est source de consolation que l'homme a à endurer de son vivant. Pour le philosophe allemand, Arthur Schopenhauer, elle

F. U. Angrey (ه)

Faculty of Arts, University of Calabar, P. M. B. 1115, Calabar, Nigeria

e-mail: fuangrey@yahoo.com 
est le "but de la vie". Quoi qui il en soit, la mort reste pour l'homme une énigme et un mystère qu'il n'est pas à même de sonder mais elle reste, aux dires d'Aire,

Une source intarissable de divers types d'inspiration, surtout dans le domaine artistique: poésie lyrique, fables, sculpture (p. 3).

Par ce quelle démontre de la mort dans les romans qui constituent le fondement de notre étude, Maryse Condé se veut une disciple invétérée de la théorie freudienne de la psychanalyse qui est la motivation vers la mort, ver l'auto-destruction et vers le retour à l'inorganique. Cette théorie, élaborée par Wilhelm Stekel dans la pensée post-freudienne, se réfère à cette motivation de la mort comme "Thanatos".

Les personnages condéens, dont Razyé dans La migration des cours, Francis Sancher dans Traversée de la mangrove, sont guidés par cette motivation de la mort. Leurs actions et inactions se font dans le but de mener à terme ce qu'ils considèrent leur "chaude-pissede vie". Ils décident, le plus souvent, de s'auto-détruire afin d'accéder à une vie supérieure par la voie de la mort.

Maryse Condé, écrivaine guadeloupéenne, fait ainsi de la mort un sujet intéressant de discussion dans certains de ses romans. Par une lecture thanaticopsychologique de ses romans, dont La vie scélérate, Les derniers rois mages (1993), Traversée de la mangrove (1989), La colonie du nouveau monde (1992), La migration des cours (1995), nous tenterons une analyse thanatico-psychologique du thème de la mort comme vu par Condé.

\section{La mort comme source de révolte}

Dans des romans de Maryse Condé, la mort d'un individu peut, de beaucoup, influencer des décisions prises par certains personnages. Elle peut faire se révolter bon nombre de personnes qui trouvent cette mort inacceptable donc de mauvais goût. Dans La vie scélérate, roman qui relate la scélératesse de la vie de misère que mène le petit peuple antillais, la mort de Mano, le père d'Albert Louis, est le déclic psychologique dont ce dernier a besoin pour changer non seulement son sort à lui mais aussi celui de toute une famille.

En effet, quand Albert Louis voit mourir Mano dans des conditions pitoyables, il se sent humilié et même offusqué. Il se décide alors à s'émigrer vers d'autres cieux où la vie, semble-t-il, lui serait meilleure. La décision qu'il prend de partir de la plantation où meurt son père est en connaissance du fait qu'il doive ostensiblement éviter de mourir dans les mêmes conditions que son père. La romancière nous rappelle:

Depuis qu'il avait vu mourir Mano, son père, le corps si décharné qu'il ne faisait pas le poids de celui d'un enfant. Les bras et les jambes déformés comme des branches de goyavier, Albert s'était juré de fuir la canne (p. 16).

Cette décision de vouloir fuir la canne marque une révolte, une répudiation, un rejet et un refus des conditions inhumaines dans lesquelles on fait travailler le petit peuple qui semble n'avoir pas de lendemain. Le départ d'Albert Louis pour le Panama, où les Américains font construire un canal, lui est de bon augure. En effet, 
il en revient plus riche et plus nanti en matière d'argent. Avec les dollars qu'il en ramène, il s'achète une maison; il réussit à faire sortir sa mère, Théodora, de l'étau de la misère et à améliorer son statut social.

La mort joue aussi un rôle très important dans les actions et dans les inactions des uns et des autres des personnages que l'on rencontre dans La migration des cœurs. D'abord, Condé essaie de démontrer que la mort est un phénomène qui ne respecte personne par le meurtre de Melchoir, le babalawo, qui "lisait l'avenir de tout un chacun" (p. 18). En tant que liseur de l'avenir, on s'attend à ce que Melchoir prédise son meurtre et l'évite.

La mort de ce quimboiseur dans des circonstances de meurtre souligne jusqu'à quel point des crimes se passent à la Havane, la capitale cubaine. Elle souligne le fait que tout Havanien vit dans la peur et dans l'incertitude de l'avenir. La romancière souligne ce fait en ces termes.

Car, jour après jour à La Havane, c'était crime sur crime. Dans tous les quartiers, les plus huppés comme les plus louches, le sang coulait. On ne pouvait plus distinguer entre assassinats politiques et assassinats crapuleux (p. 18).

C'est ce qu'au-dedans de lui-même il ressentait En dehors de cette mort, qui symbolise la violence qui sévit dans la capitale cubaine, Razyé, un des personnages principaux du roman, la considère une perte personnelle dans la mesure où il s'était rendu apprenti babalawo auprès de Melchoir. Il voit ses rêves d'être comme ce dernier s'estomper à cause de sa mort inattendue. Pour lui, Melchoir lui a joué un mauvais tour.

Fureur et exaspération, comme si la mort de Melchoir était un mauvais tour que le babalawo lui avait joué (p. 19).

Par le fait même, il ne pourra réaliser son rêve d'enfance de communiquer avec les morts, phénomène qu'il aurait auprès de son "maître".

Ainsi, il ne réaliserait jamais le rêve de son enfance et ne communiquerait jamais avec les disparus. Il ne serait jamais dans les secrets de l'invisible et il ne pourrait pas remodeler le monde à sa guise (p. 19).

Son échec imprévisible, dû à la mort de Melchoir, le rend aigre et vengeur. Désormais, il n'a qu'une intention qui est celle de retourner en Guadeloupe pour se venger de Cathy, la femme qu'il aime mais qui ne veut point de lui, et de son mari, Aymeric Linsseuil. La mort de Melchoir accentue le besoin chex lui de vouloir se venger contre ses "deux ennemis". C'est ce besoin qui empoissonne ses relations avec son entourage.

\section{La mort, élément rassembleur}

Dans les rapports qui existent souvent entre les personnages condéens, comme on le voit dans certains des romans de Condé, il est bien difficile de parler d'une certaine intégration. Ce sont de petites jalousies, la discrimination, même au sein d'un même 
groupe racial, l'égoïsme qui animent le cœur des personnages. Aux yeux de certains de ces personnages, la Guadeloupe est une île caractérisée par le durcissement des cœurs de ses habitants. Quand Razyé, par exemple, décide d'y retourner après la mort de Melchoir, son maître babalawo, Stéfania Fonéca, sa maîtresse, lui tient ce discours pour le persuader de rester à Cuba.

J'entends que vous partez pour la Guadeloupe? Qu'est-ce que vous allez chercher dans cette petite île de rien du tout où on ne parle même pas l'espagnol? Vous m'avez dit vous-même que le cœur des gens est plus dur que la pierre de silex, que l'eau de la compassion ne l'arrose jamais! (p. 21)

Cette constatation ne concerne pas seulement ce personnage de Fonséca dans $L a$ migration des cœurs. Le phénomène du durcissement des cœurs humains se retrouve aussi dans Traversée de la mangrove où les habitants de Rivière au Sel, cadre du roman, mènent une vie égoïste et xénophobe. Les relations entre les habitants restent très tendues; ceci rend malheureuse la vie de plusieurs personnes puisqu'elles vivent dans la solitude et dans l'oubli. C'est ce que nons disons quand nons faisons remarquer:

Dans traversée de la mangrove, les habitants de Rivière au Sel sont voués à la solitude. Ils vivent abandonnés et marginalisés par leur entourage... (p. 14).

Le roman de Condé privilégie ainsi les difficiles relations qui existent entre les gens qui habitent cet espace terrien où ils se soupçonnent les uns les autres car ils ne s'aiment pas. C'est sur ce que Mira, un des personnages du roman, semble se plaindre:

Les gens de Rivière au Sel ne s'aiment pas. Les femmes récitent leurs prières à la sainte Vierge quand elles croisent mon chemin. Les hommes se rappellent leurs rêves de la nuit quand ils ont trempé leurs draps et ils ont honte. Alors, ils me bravent des yeux pour cacher leur désir (p. 57).

De cette manière, l'environnement physique où se déploient ces habitants leur est très hostile puisqu'il les abrutit; il dégrade leurs mœurs. Les gens y sont très méchants; ils passent le gros de leur temps à médire des autres. Pour cette raison, certains personnages, dont Mira, fuient les yeux des hommes pour se réfugier dans la ravine.

...chaque fois que j'ai le cœur ensanglanté à cause de la méchanceté des gens de Rivière au Sel qui ne savent qu' affûter le couteau de leurs paroles de médisance, je descends à la ravine (p. 52).

La présence de Francis Sancher, un étranger né loin de "notre île à ragots; livrée aux cyclones et aux ravages de la méchanceté des cœurs des hommes" (p. 63), accentue l'attitude xénophobe de ces habitants. Personne ne veut le recevoir, personne ne veut de lui à Rivière au Sel. Chaque habitant semble lui en vouloir peut-être à cause de sa couleur blanche. L'étranger n'arrive pas non plus à s'intégrer aisément dans cette société où les gens vivent enfermés sur eux-mêmes.

Même quand Francis Sancher meurt on abandonne son cadavre dans un coin de la route. De son vivant, il n'a ni parents ni amis. Ceci étant donc le cas, on s'attend à 
ce que personne ne le pleure ni ne se soucie des arrangements visant à enterrer le mort. Mais chose curieuse une foule nombreuse assiste à sa veillée bien que ceci ne se fasse pas pour le mort.

Pendant la veillée, chacun des habitants essaie de se remémorer ses relations avec Francis Sancher. Dans le système des valeurs sociales des Antillais, la veillée du mort, comme celle de Sancher, est un rassemblement. La mort devient par le fait même un élément rassembleur très important puisque des parents, des voisins et des amis se rassemblent pour se conter des histoires, pour prendre part à certains jeux ludiques. Pour Léonard Sainville (1977),

La veillée du mort est l'objet d'un qrand rassemblement où sont présents les voisins et les amis les plus lointains, où l'on se livre à force libations et où le récit des contes comme l'étalage des devinettes tiennent une place dominante (p. 145).

La veillée mortuaire n'est donc pas seulement un fait physique. Elle devient la seule occasion où les gens de toutes les couches sociales et raciales peuvent s'intégrer, elle est par les fait même une rassembleuse de pensées et d'idées et, aux dires de Marie-Agnès Sourieau (1995), "un champ de pulsions communes." On assiste à la veillée pas nécessairement pour le mort. On le fait pour avoir une de ces rares occasions, offertes par la veillée, d'exprimer ses peurs, ses troubles, de s'ouvrir aux autres et ainsi de se soustraire, ne serait-ce que pour un instant, à la psychose générale où semble sombrer toute la communauté.

Suivant le même raisonnement que Sourieau, Mitsch trouve que la veillé n'est qu'un moyen propice pour les membres de la petite communauté riviérienne de tous âges de se concerter et de revoir ensemble leurs relations avec autrui (2012). C'est aussi le moment, d'une seule nuit, de se remémorer leur passé et, surtout, de considérer leurs espoirs d'avenir. Mitsch est d'avis que

Citizens of all ages and of all social strata come together in one space for one night, bringing their memories of the past as well as their hopes for their own future lives, all of which devolve from their association with this one man (p. 55).

Ceci démontre que les habitants qui, d'ordinaire, ne s'assemblent pas, voient la veillée, conséquence de la mort, comme un appel à une réunion inopinée.

\section{La mort ou la quête de la paix}

Dans notre discussion de la mort jusqu'ici, nous n'avons pas cherché à savoir pourquoi on meurt, ce qui fait mourir ou des conditions dans lesquelles on meurt. Bien que la mort soit un phénomène naturel qui doit venir à l'homme soit avec l'âge soit encore avec la maladie, il est des moments où l'homme préfère la mort à la vie. Dans un moment de délire, dû à la frustration, il arrive que l'homme se décide à mourir pour ainsi retrouver la paix intérieure.

Bon nombre d'écrivains ont su poétiser ce phénomène dans leurs écrits. Pour Baudelaire, par exemple, “c'est la mort qui console...et qui fait vivre” (p. 452) 
surtout pour les "pauvres" qui ont besoin d'elle pour mettre fin à leur vie de misère. Par la mort, ils "recevront le prix de leurs misères" (p. 452).

Dans les romans de Condé, certains personnages contemplent la mort, souvent par le suicide, quand ils se sentent délaissés ou mal aimés par ceux qu'ils croient aimer. Dans La migration des cœurs, Razyé trouve qu'il n'a plus raison de vivre une fois que Cathy, la femme qu'il croit aimer, lui tourne le dos. Pour lui, la vie ne vaut plus la peine d'être vécue.

Pourquoi continuait-il à marcher sur le chemin des vivants? Celle qui étant plus que sa vie même lui avait tourné la dos. Il lui semblait que ses protestations méprisantes n'avaient cessé de se répercuter en écho dans sa vie (p. 20).

Razyé se sent ainsi humilié par cette situation qu'il considère désobligeante et dégradante. Le rejet et l'abandon dont il est victime deviennent pour lui un poison dont il se sert pour infecter la vie des gens dans son entourage. Quand il fait irruption dans le domaine des Belles-Feuilles toute la maisonnée sent que "le malheur vient d'entrer dans cette maison" (p. 63).

Dans le domaine où se trouve désormais Razyé les relations entre les membres de la maisonnée deviennent très tendues. Il se crée des tensions non seulement dans la maison mais aussi dans le cœur des hommes. Les conflits internes entre les membres de la maisonnée commencent à influer négativement sur leur santé et même sur leurs comportements.

Cathy, l'amante de Razyé et la femme d'Aymeric Linsseuil, commence à perdre du poids, elle ne connaît plus la joie de vivre. Elle devient, par le fait même, une femme ratatinée et méconnaissable pour qui le mari fuit la maison. Ce dernier ne peut plus supporter sa compagnie.

Le maître, lui était parti à l'usine et n'était pas près de réapparaître. Il s'absentait comme cela toute la journée...il ne pouvait pas supporter la compagnie de sa femme, voir le constrate entre ce qu'elle avait été et ce qu'elle était devenue (p. 85).

Sur ces entrefaites, il n'est pas surprenant que Cathy choisisse le chemin de la mort. En effet, elle se “donne" la mort pour mettre fin à la vie de honte qu'elle est appelée à mener partagée entre un amant et un mari.

Après la mort de cette jeune femme, qui n'a connu que la misère de son vivant, la rivalité entre Razyé et Aymeric ne s'apaise pas. Razyé continue de s'embarquer sur des activités négatives qui ruinent Aymeric matériellement et physiquement. Ces activités poussent les gens à la mort. Poussé par la jalousie et par le goût de la vengeance, Razyé fait détruire et fait brûler toutes les plantations de canne appartenant à Aymeric Linsseuil. Tous les noirs et tous les Indiens qui travaillent pour le compte de ce dernier se retrouvent sans emplois. Plusieurs familles sont ainsi plongées dans la crasse de la misère et dans la pénurie.

N'y tenant plus, Aymeric meurt comme pour se soustraire à la misère causée délibérément par Razyé que la dureté de cœur et la méchanceté poussent à faire des gestes insensés. Mais après la mort prématurée d'Aymeric, Razyé prend conscience des faits et gestes des uns et des autres. Avec un examen suivi de sa conscience, il se 
sent responsable de toutes les morts et de toutes les souffrances dont sont victimes les membres du domaine des Belles-Feuilles et de toute la communauté. Il reconnaît son forfait pour lequel il ne craint pas d'aller brûler éternellement en enfer, qu'il porte déjà en lui.

L'enfer, il le portait en lui, à l'emplacement du cœur. C'est qu'il était un lâche, voilà tout. Il n'avait pas le courage de devancer son temps. Où, alors, c'est qu'il était un hypocrite...mais, à présent, il ne tenait plus à rien (pp. 253-254).

Il ne tient plus à rien puisqu'il a accompli la mission de sa vengeance qui est celle de tuer. Il perd le goût de la vie n'ayant plus rien à gagner d'elle l'objet de sa passion aveugle, Aymeric, ayant passé. Tout autour de lui commence à l'ennuyer. Il souhaite plutôt qu'il meurt pour mettre fin à la vie de chien qu'il mène désormais.

D'ailleurs tout l'ennuyait en ce moment. Marcher. S'habiller. Boire du Rhum. Faire l'amour. Il n'avait goût à rien...Depuis qu'Aymeric avait passé, la vengeance qu'il avait poursuivie n'avait plus de sens, et il ne voyait plus d'objet à sa vie (p. 247).

En fin de compte, Razyé devient une épave humaine qui ne peut plus répondre de ses actes. Il finit par mourir torturé et hanté par ses péchés et par la vie de misère qu'il fait mener à son entourage.

Dans La colonie du nouveau monde, où "Maryse Condé raconte la bouleversante déréliction d'une communauté d'hommes et de femmes revenus à l'adoration des divinités d'autrefois", la mort est aussi conçue comme une belle façon de mettre fin à la "chaude-pisse de vie" que l'on mène. En effet, La Ceja, la communauté d'Antillais qui se trouvent en Colombie, devient une ville dangereuse prise d'assaut par le vol et le viol, la promiscuité sexuelle.

Dans des conditions sociales pareilles, la débandade de la colonie semble désormais consommée. Elle se trouve décimée et ses membres se sentent par la suite abandonnés non seulement par les hommes mais également par Dieu. La Ceja devient par le fait même une "confrérie des abandonnés" pour qui la vie n'est que misère, pénurie et carence de tout ce dont on a besoin pour mener une vie acceptable.

Pour empirer la situation déjà désespérée de ces abandonnés, les autorités municipales font promulguer un arrête d'expulsion qui veut que ceux-ci déguerpissent leur colonie dans un mois. La question "pour aller où?" préoccupe désormais ces habitants. Rudolf, un de ces abandonnés, se décide à y rester pour attendre que la mort le prenne. De son vivant, il ne connaît que déception et chute. Par la mort, il pourrait en finir avec la misère.

Plus pour lui! Il l'avait compris, sur cette terre, il n'y a pas de Terre promise. Quel que soit l'endroit vers lequel nous voyageons, nous nous retrouvons identiques à nous-mêmes avec nos peurs et nos fatalités. Il resterait là, à La Ceja, replié sur lui-même en attendant que la mort vienne à son secours (pp. 236-237).

Ce qui est encore plus intéressant dans le roman est que certains personnages dont Aton, Rudolf, Mandjet, commettent un suicide collectif. Ils se font tous brûler 
par un feu qu'allume Aton. La raison de ce suicide collectif est toute simple: se soustraire à la souffrance qu'offre la vie sur cette terre comme le dit la romancière à propos de Mandjet:

De quoi avait-elle peur? Le visible que nous connaissons n'est-il pas plus effrayant que l'invisible que nous ignorons? Pourrait-elle souffrir dans l'audelà plus qu'elle n'avait déjà souffert sur la terre? (p. 244)

Cette question, à laquelle elle ne saurait elle-même répondre, marque le paroxysme du désespoir dans lequel elle est plongée. Elle montre aussi que les membres de cette communauté sont tous des hommes désespérés qui préfèrent la mort à la vie de misère qu'ils mènent. La seule rescapée du suicide collectif qu'ils se donnent est Méritaton, fille d'Aton, qui doit retourner en Guadeloupe avec le rêve de cette colonie qui est, pour elle,

L'endroit où elle avait été initiée à la fois à la maladie, à la souffrance, à la cruauté et à la mort. Où qu'elle aille, quoi qu'elle fasse, elle ne pourrait jamais rejeter ces jours de sa mémoire (p. 249).

Dans Les derniers rois mages, Spéro, abandonné par sa femme, Debbie, et par sa fille, Anita, se sent seul et rejeté. Du retour d'Amérique où il n'est qu'un fainéant, il mène une vie à la dérive en Martinique. Dans un excès de désespoir et d'angoisse, il contemple aussi la mort, échappatoire à toutes ses souffrances.

Tout seul sur cette jetée déserte, Spéro retrouvait la peur et l'angoisse de ses douze ans. La nuit et sa couleur d'encre de chine est l'image de la mort (p. 303).

L'image que Spéro se fait de la mort est celle de la paix et de la douceur, surtout pour un homme comme lui qui a toujours sombré dans la fainéantise et ainsi n'a pas su faire face aux combats de la vie. C'est en ces mots qu'il explique le phénomène:

Peut-être au contraire est-ce le lieu de la paix, de la fin de tous les combats?

Qu'est-ce que la mort? Un voile de crêpe noir que l'on étend doucement, tout doucement sur les yeux et qui assourdit toutes les souffrances (p. 303).

Bien que Spéro conçoive ainsi la mort, il n'a pas le courage de se la donner comme l'ont fait les "abandonnés" de La Ceja dans La colonie du nouveau monde. Il préfère vivre d'espoir car, pour lui, “à force d'espérance et de patience, il finirait à nouveau par rencontrer Debbie” (p. 304).

\section{Conclusion}

Dans notre communication, nous avons pu démontrer que la mort, selon Condé, est une "migration sans retour". En faisant cela, nous avons pris en compte les différentes manifestations de cette énigme qu'est la mort dans certains romans de l'écrivaine guadeloupéenne. Bien que ses romans ne fassent pas preuve "d'une obsession thanatique voisine de la nécrophilie" (p. 127), ils mettent en lumière le thème de la mort. 
Dans ce travail, nous avons essayé de discuter de ce thème comme source importante de révolte dans la mesure où la mort de certains personnages poussent d'autres personnages à se révolter contre une situation qu'ils trouvent désobligeante et, donc, de mauvais goût. En nous servant de La vie scélérate et de La migration des cœurs nous avons pu démontrer ce phénomène.

Condé essaie également de nous montrer que la mort peut être un élément rassembleur à plusieurs égards. La veillée du mort devient ainsi le moment pendant lequel des gens d'une communauté peuvent se réunir et discuter des problèmes qui se posent aux individus et à la communauté tout entière. C'est ce que nous faisons ressortir de Traversée de la mangrove qui est un "champ de plusions communes", surtout quand on sait qu'à Rivière au Sel, cadre privilégié du roman, les gens ne "s'aiment pas".

En tout et pour tout, la mort est souvent quête de la paix. Par elle, on arrive à mettre fin à la misère et à la souffrance. Or, dans les romans de Condé, nous sommes toujours face à des hommes complexes et complexés qui n'arrivent pas à supporter la vie de misère qu'ils sont appelés à vivre. N'y pouvant tenir, ils cherchent la voie de la mort pour y mettre fin. Nous nous somme référé à des personnages comme Cathy, Aymeric Linsseuil et Razyé dans La migration des coeurs, Aton, Mandjet et Rudolf dans La colonie du nouveau monde, qui se donnent la mort par un suicide collectif, Spéro dans Les derniers rois mages qui manque de courage de se suicider car il espère que sa condition pourrait évoluer.

Par une lecture thanatico-psychologique, nous avons pu démontrer l'importance de la mort dans des romans de Condé bien que l'accent ne soit pas mis sur son "goût morbide de la mort" (p. 127). Nous n'y avons pas essayé de montrer que la romancière s'adonne à la dialectique vie/mort. Tout ce qu'elle fait, et ce que nous avons pu démontrer, c'est qu'elle juxtapose le phénomène de la mort à la misère et à la souffrance qui accablent l'homme et font de lui un jouet dans les mains de la mort.

Open Access This article is distributed under the terms of the Creative Commons Attribution License which permits any use, distribution, and reproduction in any medium, provided the original author(s) and the source are credited.

\section{Bibliographie}

Aire, V. (2004). Thanatos and Eros: Death in life and in French literature. Inaugural lecture. Jos: University of Jos.

Aire, V. (2005). Essais sur le roman francophone africain. Bookhouse: Jos St. Stephen INC.

Angrey, F. U. (1998). Le projet idéologique de Maryse Condé dans La vie scélérate. In S. Ade Ojo (Ed.) RENEF; $1 / 6$ (pp. 86-103). Lagos: SES.

Angrey, F. U. (2005). Misère chez soi, quête du bonheur chez l'autre: mouvance, salut et contradictions dans les romans de Maryse Condé. Synergies Afrique Australe, 1, 84-91.

Angrey, F. U. (2006). Mouvance, quête identitaire et peur du phénomène de la mondialisation dans la littérature: exemple de Maryse Condé. Neohelicon, 33(1), 231-238.

Angrey, F. U. (2008). Solitude et tristesse, produits de l'environnement humain: Un nouveau regard sur Traversée de la mangrove de Maryse Condé. La Revue de Études Francophone de Calabar, 7(1), $121-135$.

Angrey, F. U., \& Akpagu, Z. I. (2003). L'univers imaginaire de Maryse Condé et l'intégration sociale et raciale comme condition sine qua non pour une légitimation identitaire aux Antilles. Ndunode: Calabar Journal of the Humanities, 1, 12-23. 
Balutansky, K. (1995). Créolité in question: Caliban in Maryse Condé's Traversée de la mangrove. In M. Condé \& M. Cottenet-Hage (Eds.), Penser la créolité (pp. 101-111). Paris: Karthala.

Carruggi, N. (Ed.). (2010). Maryse Condé: Rébellion et transgression. Paris: Karthala.

Chamoiseau, P. (1991). Reflections on Maryse Condés Traversée de la mangrove. Callaloo, 142, 389-395.

Condé, M. (1987). La vie Scélérate. Paris: Editions Seghers.

Condé, M. (1989). Traversée de la mangrove. Paris: Mercure de France.

Condé, M. (1992). La colonie du nouveau monde. Paris: Robert Laffont.

Condé, M. (1993). Les derniers rois mages. Paris: Robert Laffont.

Condé, M. (1995). La migration des cœurs. Paris: Robert Laffont.

Condé, M., \& Cottenet-Hage, M. (Eds.). (1995). Penser la créolité. Paris: Karthala.

Cottenet-Hage, M., \& Moudelino, L. (Eds.). (2002). Maryse Condé: une nomade inconvenante. Jarry (Guadeloupe): Ibis Rouge.

Fischer, G.-N. (2005). Les concepts fondamentaux de la psychologie sociale. Paris: Dunod.

Hess, D. (2011). Maryse Condé: mythe, parabole et complexité. Paris: L'Harmattan.

Lagarde, A., \& Michard, L. (1985). XIX $X^{e}$ siècle: Les grands auteurs du programme. Paris: Bordas.

Lionnet, F. (1993). Traversée de la mangrove: vers un nouvel humanisme. French Review, 66(3), 475-486.

Lucey, M. (1992). Voices accounting for the past: Maryse Condé's Traversée de la mangrove. In M. Condé (Ed.), L'héritage de Caliban (pp. 123-132). Pointe-à-Pitre: Editions Jasor.

Mitsch, R. H. (2012). Maryse Condé's mangroves. Accessed August 9, 2012, from www.hu.ntu.edu.

Mokwenye, C. O. (2011). Black literature as testimony: The francophone Caribbean's quest for his African identity; inaugural lecture. Nigeria: University of Benin.

Perret, D. (1992). L'écriture mosaïque de Traversée de la mangrove. In M. Condé (Ed.), L'héritage de Caliban (pp. 179-190). Pointe-à-Pitre: Editions Jasor.

Pfaff, F. (1993). Entretions avec Maryse Condé. Paris: Karthala.

Sainville, L. (1977). Les fondements négro-africains de la culture dans les Caraïbes et la lutte pour leur sauvegarde. Présence Africaine, 101-102, 129-157.

Sourieau, M. A. (1995). La vie Scélérate de Maryse Condé: métissage narratif et héritage métis. In M. Condé \& M. Cottenet-Hage (Eds.), Penser la Créolité. Paris: Karthala. 